\title{
Dynamical origin of spontaneous symmetry breaking in a field-driven nonequilibrium system
}

\author{
R. D. Willmann ${ }^{1}$, G. M. Schütz ${ }^{1}$ and S. Grosskinsky ${ }^{2}$ \\ 1 Institut für Festkörperforschung, Forschungszentrum Jülich, 52425 Jülich, Germany \\ 2 Zentrum Mathematik, TU München, 85747 Garching bei München, Germany
}

PACS. 05.70.Ln - Nonequilibrium and irreversible thermodynamics.
PACS. 02.50.Ey - Stochastic Processes.
PACS. 64.75.+g - Phase separation.

\begin{abstract}
A one-dimensional driven two-species model with parallel sublattice update and open boundaries is considered. Although the microscopic many-body dynamics is symmetric with respect to the two species and interactions are short-ranged, there is a region in parameter space with broken symmetry in the steady state. The sublattice update is deterministic in the bulk and allows for a detailed analysis of the relaxation dynamics, so that symmetry breaking can be shown to be the result of an amplification mechanism of fluctuations. In contrast to previously considered models, this leads to a proof for spontaneous symmetry breaking which is valid throughout the whole region in parameter space with a symmetry broken steady state.
\end{abstract}

While single-species driven diffusive systems in one dimension are largely understood, twospecies models show a variety of phenomena that are a matter of current research, such as phase separation and spontaneous symmetry breaking (see 1 for a recent review). The first such model that was shown to exhibit spontaneous symmetry breaking was a model with open boundaries that became known as the 'bridge model' 2]. In this model, two species of particles move in opposite directions. Although the dynamical rules are symmetric with respect to the two species, two phases with non-symmetrical steady states were found by Monte Carlo simulations and mean-field calculations. While the existence of one of the phases remains disputed [3,4], a proof for the existence of the other one was given for the case of one vanishing boundary rate [5]. Recently, a variant of the bridge model with non-conserving bulk dynamics was considered [6]. Although the phase diagram of this model is even richer than that of the original one, a proof for a symmetry broken state could again only be given in the case of one vanishing boundary rate. Since spontaneous symmetry breaking does not occur in one-dimensional systems in thermal equilibrium and since not even a macroscopic description in terms of boundary reservoirs is known [7,8] it would be desirable to gain insight in the dynamical origin of this genuinely nonequilibrium phenomenon.

The symmetry breaking models considered so far evolve by random sequential update. In this article, a variation of the bridge model with parallel sublattice update is studied. The update scheme ensures that the dynamics in the bulk is deterministic, while stochastic events occur at the boundaries. Thus - while maintaining noisy dynamics - the complexity of the 
problem is reduced, which allows to elucidate the mechanism by which spontaneous symmetry breaking occurs in this model as well as to give a proof for the existence of a symmetry broken phase. This proof is valid for the whole region in parameter space where symmetry breaking occurs and not just in some limiting case.

The model considered here is defined on a one-dimensional lattice of length $L$, where $L$ is an even number. Sites are either empty or occupied by a single particle of either species $A$ or $B$, i.e., the particles are subject to an exclusion interaction. The dynamics is defined as a parallel sublattice update scheme in two half steps. In the first half-step the following processes take place: At site 1 it is attempted to create a particle of species $A$ with probability $\alpha$ if the site is empty, or to annihilate a particle of species $B$ with probability $\beta$, provided the site is occupied by such a particle:

$$
0 \stackrel{\alpha}{\rightarrow} A \quad B \stackrel{\beta}{\rightarrow} 0
$$

At site $L$, a particle of species $B$ is created with probability $\alpha$ and a particle of species $A$ is annihilated with probability $\beta$ :

$$
0 \stackrel{\alpha}{\rightarrow} B \quad A \stackrel{\beta}{\rightarrow} 0 .
$$

In the bulk, the following hopping processes occur deterministically between sites $2 i$ and $2 i+1$ with $0<i<L / 2$ :

$$
A 0 \rightarrow 0 A \quad 0 B \rightarrow B 0 \quad A B \rightarrow B A .
$$

In the second half-step, these deterministic hopping processes take place between sites $2 i-1$ and $2 i$ with $0<i \leq L / 2$. Note that the dynamics is symmetric with respect to the two particles species. The original bridge model [2] arises as the continuous-time limit of this model with stochastic hopping.

The stationary phase diagram of the model in terms of the parameters $\alpha$ and $\beta$ can be explored by Monte Carlo simulations. Two phases are found (see Figure 1):

- If $\alpha<\beta$, the system exhibits a symmetric steady state. Here, the bulk densities are $\rho_{A}(i)=0, \rho_{B}(i)=\alpha \beta /(\alpha+\beta)$ if $i$ is odd, and $\rho_{A}(i)=\alpha \beta /(\alpha+\beta), \rho_{B}=0$ if $i$ is even.

- If $\alpha>\beta$, the system resides in the symmetry broken phase. Assume the $A$ particles to be in the majority. Then, the bulk densities in the steady state are $\rho_{B}(i)=0$ for all $i, \rho_{A}(i)=1$ for $i$ even and $\rho_{A}(i)=1-\beta$ for $i$ odd. This means that the symmetry is maximally broken and the minority species is completely expelled from the system.

- The behavior on the transition line for $\alpha=\beta$ is described below.

Thus, the dynamics of the majority species in the broken phase is identical to the single species ASEP with parallel sublattice update. For this system, the exact steady state density profile in a finite system is known [9]. The density profile of the majority species in the broken phase of the sublattice bridge model equals that of the high density phase in the sublattice ASEP at the given parameters $\alpha$ and $\beta$.

In the following, the dynamics leading to symmetry breaking is elucidated and the respective time scales are determined. For $\alpha>\beta$ an amplification mechanism of fluctuations leading to a symmetry broken state is identified. This mechanism shows that the system needs a time $T_{1}$ which is only algebraically increasing with $L$ to enter the broken phase, if it was started with symmetric initial conditions. Furthermore, assuming the system to be in the broken phase it is shown that it takes a time $T_{2}$ that is exponentially increasing with $L$ until particles of the minority species can penetrate the system. Both facts together provide a proof for spontaneous symmetry breaking in this model for $\alpha>\beta$. 

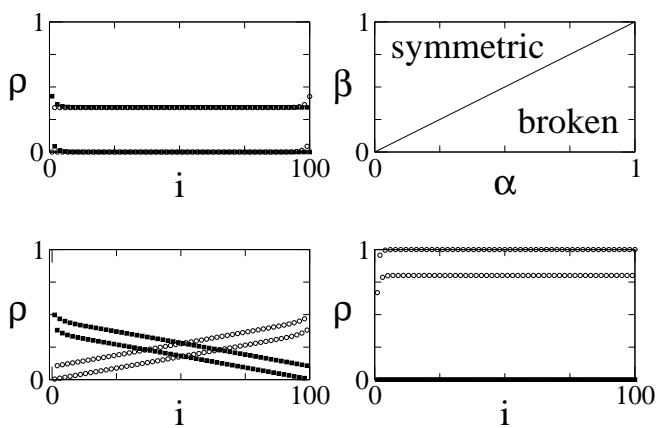

Fig. 1 - Upper right: Stationary phase diagram of the sublattice bridge model. Average density profiles as obtained from Monte Carlo simulations in the symmetric phase at $\alpha=0.6$ and $\beta=0.8$ (upper left), the broken phase at $\alpha=0.6$ and $\beta=0.2$ (lower right) and on the transition line at $\alpha=\beta=0.2$ (lower left). $A$ densities are shown by $\circ$ and $B$ densities by

Dynamics of symmetry breaking: It is assumed that at $t=0$ there are no particles in the system and that $\alpha>\beta>0$. The case of other initial densities can be treated in a similar fashion and the case $\beta=0$ leads to a degenerate situation with many steady states depending on the initial conditions, since no particle can leave the system. This is straightforward to analyze and is not discussed in the following. Starting from the empty lattice, $A(B)$ particles are created at every time step with probability $\alpha$ at site $1(L)$. Once injected, particles move deterministically with velocity $2(-2)$. Therefore, at time $t=L / 2$ the system is in a state where the density of $A(B)$ particles is $\alpha(0)$ at all even sites and $0(\alpha)$ at all odd sites. In this situation both creation and annihilation of particles are possible.

However, it turns out that the effect of creation of particles is negligible: Since $\alpha>\beta$ the deterministic hopping with velocity 2 transports on average more $A$-particles towards site $L$ than can be annihilated there. This leads to the formation of an $A$-particle jam at the right boundary, blocking the injection of $B$-particles. An analogous argument holds for the left boundary, which is blocked by a $B$-particle jam. In these jams, the only source of vacancies is annihilation at the boundaries with probability $\beta$ in the first half-step. In the second half-step the vacancy moves one site towards the bulk with probability 1 . Therefore, in a jam, the density of $A(B)$ particles at even (odd) sites is 1 , while that at odd (even) sites is $1-\beta$. So the only way to create particles in this situation is a complete dissolution of a jam. But as long as it gains particles from the low density region this is a very rare event since $\alpha>\beta$. It can be shown that the average number of created particles is small and bounded independent of $L$ [10]. So creation of particles in this jammed situation becomes negligible in the limit $L \rightarrow \infty$ and will be neglected in the following.

The number of particles in each of the two jams reduces by one in every time step with probability $\beta$. Since creation of particles is negligible, the influx into the jam ceases after some time and the jam eventually dissolves. By fluctuations, one of the jams, say the $B$-jam at the left boundary, dissolves first. $A$ particles can enter the system while $B$ particles are still blocked until the $A$-jam at the right boundary is also dissolved. The configuration of the system at this point is illustrated in Figure 2 $\left(t_{1}\right)$. The light grey region denotes a region of low density of $A$ particles where the density is $\alpha(0)$ on even (odd) sites. The (random) length of this region, $\Delta \ell_{1}$, describes the majority of one of the species. The description is symmetric, so if $\Delta \ell_{1}<0$ this corresponds to a majority of $B$ particles. Thus the average 

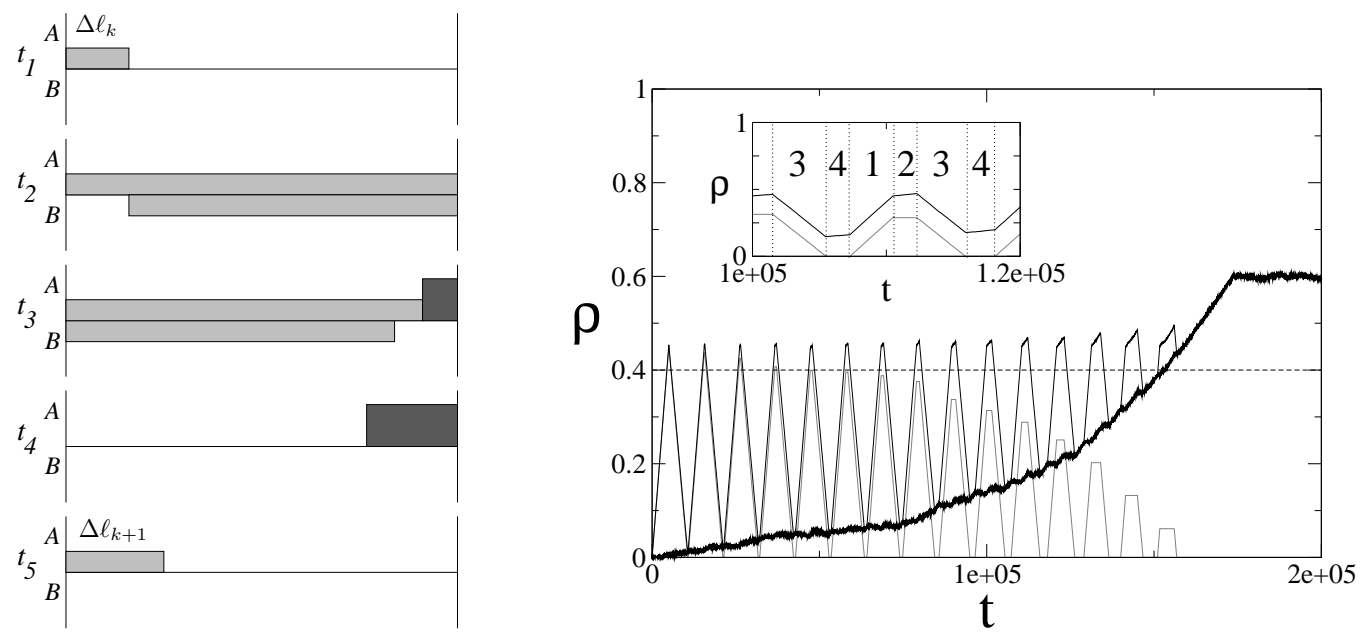

Fig. 2 - Left: Illustration of the stages involved in the $k$-th cycle of the dynamics of spontaneous symmetry breaking as explained in the text. Low density regions are drawn light grey, jams are dark grey and white regions of the system are empty.

Right: Symmetry breaking, starting from the empty lattice (single realization of MC simulation). Here, $\alpha=0.9, \beta=0.8$ and $L=10000$. The density of $A$ particles is drawn in black, that of $B$ particles in grey and the difference as the thick black line. The inset shows a close-up during the time evolution. Individual stages as described in the text are separated by dotted vertical lines.

value $\left\langle\Delta \ell_{1}\right\rangle=0$, but typically $\Delta \ell_{1}=O(\sqrt{L})$ due to Gaussian fluctuations for large $L$ and one of the species has the majority, which we assume to be species $A$.

The time evolution just described constitutes the first loop of a cyclic behaviour which can be effectively described by the dynamics of low density regions and jams at the boundaries. The key ingredient for this simplification is the jamming mechanism described above. The cyclic behaviour consists of 4 stages, which we summarize in the following and which is illustrated in Figure 2.

1. At the beginning of a cycle $\left(t_{1}=0\right)$ there is a low density region of $A$ particles at the left boundary of length $\Delta \ell_{k}$. Both species enter the system with probability $\alpha$ and penetrate the bulk deterministically with speed $2(-2)$.

2. The region of $A$ particles reaches the right boundary at time $t_{2}=\left(L-\Delta \ell_{k}\right) / 2$, creation of $B$ particles is blocked. $A$ particles still enter with probability $\alpha$ and exit with probability $\beta<\alpha$, further increasing their majority.

3. At time $t_{3}=L / 2$ the $B$ particles reach the left boundary, blocking also the creation of $A$ particles. Both species form jams at the boundaries, which gain particles from the low density regions. Since creation of particles at the boundaries is negligible, both jams eventually dissolve.

4. Let $t_{4} \geq t_{3}$ be the time when the jam of $B$ particles is dissolved and $A$ particles start to enter the system. Again, since $\alpha>\beta$ the majority of $A$ particles increases on average.

5. At time $t_{5} \geq t_{3}$ the $A$-jam at the right boundary is dissolved and also $B$ particles can enter the system. 
The cycle is finished when both jams are dissolved. In Figure 2 it is assumed that the $B$-jam dissolves first, i.e. $t_{4}<t_{5}$, which is most likely if $\Delta \ell_{k}>0$. But in general $t_{4} \geq t_{5}$ is also possible and included in the above description. The result of the cycle is

$$
\Delta \ell_{k+1}=2\left(t_{5}-t_{4}\right)
$$

which is the initial condition for the next loop. If $\Delta \ell_{k+1}<0, A$ and $B$ particles have to be interchanged for the next cycle in the above description. Note that within this framework the process starting from the empty lattice is a cycle with initial condition $\Delta \ell_{0}=0$ and $t_{2}=t_{3}$.

In the following we analyze the distributions of the random variables $t_{4}$ and $t_{5}$ to get the time evolution of $\Delta \ell_{k}$. Let $\tau_{n}$ be the (random) time it takes for a jam of length $n$ to dissolve. With this

$$
t_{4}=L / 2+\tau_{N_{B}}, \quad t_{5}=\left(L-\Delta \ell_{k}\right) / 2+\tau_{N_{A}},
$$

where $N_{A}\left(N_{B}\right)$ denotes the number of $A(B)$ particles that entered the system up to time $t_{3}\left(t_{2}\right)$ before blocking, including $\Delta \ell_{k}$. These are Bernoulli random variables with hitting probability $\alpha$, and thus the average values are

$$
\begin{aligned}
& \left\langle N_{A}\right\rangle=\left(\Delta \ell_{k} / 2+t_{3}\right) \alpha=\left(L+\Delta \ell_{k}\right) \alpha / 2, \\
& \left\langle N_{B}\right\rangle=t_{2} \alpha=\left(L-\Delta \ell_{k}\right) \alpha / 2 .
\end{aligned}
$$

As the boundary site in a jam is always occupied and particles are annihilated with probability $\beta$, the time $\tau \in\{1,2, \ldots\}$ for a particle to leave the jam is a geometric random variable with parameter $\beta$. It is $\langle\tau\rangle=1 / \beta$ and thus the average value for $n$ particles to leave is $\left\langle\tau_{n}\right\rangle=n / \beta$. So dividing (6) by $\beta$ yields $\left\langle\tau_{N_{A}}\right\rangle$ and $\left\langle\tau_{N_{B}}\right\rangle$. Using this and (4) to (6) the average value of $\Delta \ell_{k+1}$ conditioned on $\Delta \ell_{k}$ can be computed, and

$$
\left\langle\Delta \ell_{k+1}-\Delta \ell_{k} \mid \Delta \ell_{k}\right\rangle=2\left(\frac{\alpha}{\beta}-1\right) \Delta \ell_{k}+O(1) .
$$

Here the correction terms due to rare fluctuations in the boundary jams are included. They are shown to be $O(1)$ in a rigorous treatment [10, whereas $\Delta \ell_{k}$ is typically of order $\sqrt{L}$ or larger. With (7), conditioned on the initial fluctuation of $\Delta \ell_{1}$, the average value after $k$ loops is

$$
\left\langle\Delta \ell_{k+1} \mid \Delta \ell_{1}\right\rangle=\left(\Delta \ell_{1}+O(1)\right)\left(2 \frac{\alpha}{\beta}-1\right)^{k} .
$$

As $q=\left(2 \frac{\alpha}{\beta}-1\right)>1$ this constitutes an amplification of the initial fluctuations $\Delta \ell_{1}=O(\sqrt{L})$.

$\Delta \ell_{k}$ can be interpreted as a random walker on $\{-L, \ldots, L\}$. The jump probabilities are in principle given with (4) and (5) but such a detailed (and cumbersome) analysis is not necessary. Since $\alpha>\beta$ the walker is driven towards the boundaries with drift (7) proportional to $\Delta \ell_{k}$. As long as $-L<\Delta \ell_{k+1}<L$ the cycle can restart with $0<t_{2} \leq t_{3}$, and all the stages are well defined within a rigorous treatment [10. Thus initial fluctuations of $\Delta \ell_{1}=O(\sqrt{L})$ are amplified, and when $\left|\Delta \ell_{k}\right|$ reaches system size the amplification loop stops. In this situation, the effect of injection of $B$-particles is negligible for large $L$ due to jamming of $A$-particles at the right boundary. The only relevant stochastic boundary processes are injection of $A$-particles at site 1 and annihilation of $A$ at site $L$. As there is a constant supply of $A$ particles the jam quickly fills the system within a time of order $L$, reaching a state as for the single-species ASEP [9] with sublattice update at the given $\alpha, \beta$.

Time to reach the broken state: With (8) it is

$$
\left\langle\Delta \ell_{k+1} \mid \Delta \ell_{1}\right\rangle \geq L \quad \text { if } \quad k \geq \frac{\ln L-\ln \left(\Delta \ell_{1}+O(1)\right)}{\ln q}=O(\ln L) .
$$


and we denote by $\bar{k}$ the number of cycles until (9) for the average value is fulfilled. But in general the number of cycles until the amplification loop ends is a random variable. Analogous to (7) one can get a recursion relation for the standard deviation $\sigma\left(\Delta \ell_{k}\right)$ [10] and show that $\sigma\left(\Delta \ell_{\bar{k}}\right)=o(L)$. Thus fluctuation are small compared to the average value, and for $L \rightarrow \infty$ one has the expansion

$$
\frac{\Delta \ell_{\bar{k}}}{L}=\frac{\left\langle\Delta \ell_{\bar{k}}\right\rangle}{L}+\xi \frac{\sigma\left(\Delta \ell_{\bar{k}}\right)}{L}=1+\xi o(1)
$$

for almost every realization of the process, where $\xi$ is a random variable of order 1 . In turn the average number of cycles to reach the end of the amplification loop is $\bar{k}$ as determined by condition (9), and moreover, fluctuations vanish in the limit $L \rightarrow \infty$.

The average length of a loop is given by

$$
\left\langle t_{5}\right\rangle=\left(\frac{\alpha}{\beta}+1\right) \frac{L}{2}+\left(\frac{\alpha}{\beta}-1\right)\left\langle\Delta \ell_{k} \mid \Delta \ell_{1}\right\rangle / 2=O(L) .
$$

The second term depending on the index $k$ is negligible unless for $k$ close to $\bar{k}$, and even then the prefactor is smaller then the one of the first term. Moreover, since $t_{5}$ is given by a sum of independent random variables, fluctuations are Gaussian of order $\sqrt{L}$ for large $L$, and thus the length of a loop is almost constant, independent of $k$. This is confirmed in Figure 2 where a single realization of the process is plotted. Since (10) is also conditioned on the initial fluctuation $\Delta \ell_{1}$, fluctuations of this quantity constitute the only source of randomness in the time evolution for large $L$.

In total, the average time to reach the symmetry broken phase is typically $T_{1}=O(L \ln L)$. Thus, by amplification of fluctuations the system reaches a state of broken symmetry in a time algebraically increasing with $L$, provided that $\alpha>\beta$. The broken states that are attained are the steady states of the single species ASEP at the respective $\alpha, \beta$. These states either contain only $A$ - or $B$-particles, depending on initial fluctuations.

Residence time in the broken state: Assume the system to be residing in the broken state with particle species $A$ in the majority. For $L \rightarrow \infty$ this means $\rho_{A}(i)=1+o(1)$ for even sites and $\rho_{A}(i)=1-\beta+o(1)$ for odd sites, up to boundary effects at the left boundary with $i=O(1)$. Species $B$ is expelled from the system and injection of $B$ particles is only possible if site $L$ is empty. Exact expressions given in [9] (equation (18)) yield

$$
\rho_{A}(L)=1-\left(1-\frac{\beta(1-\alpha)}{\alpha(1-\beta)}\right)\left(\frac{\beta}{\alpha}\right)^{L} .
$$

Thus the probability that site $L$ is empty is exponentially small in the system size, and the time $T_{2}$ until the minority species can penetrate a system started in the broken state is exponentially large in $L$. This is not surprising even without knowledge of the exact expressions, since for injection of the first $B$ particle the complete jam of $A$ particles has to be dissolved against the drive $\alpha>\beta$. This jam consists of the order of $L$ particles. Together with the statement about $T_{1}$ from above, this proves spontaneous symmetry breaking.

The amplification mechanism outlined above does not apply for $\alpha<\beta$ since the formation of boundary jams, a key ingredient for the amplification mechanism, does not work. The length of a boundary jam is driven towards small values so the boundary sites are not blocked and particles are injected all the time.

Dynamics on the transition line: For the borderline case $\alpha=\beta$ the end of a boundary jam is diffusing and can take very large values. So the cyclic behaviour can still be observed, but 
fluctuations are larger and the cycle lengths, though still of order $L$, are strongly fluctuating. But even if this effective description is still valid, according to (7) there is no amplification of fluctuations during a cycle. Instead, $\Delta \ell_{k}$ is not driven towards the boundary but is diffusing, so a symmetry broken state can still be reached within $O\left(L^{2}\right)$ cycles, and thus $T_{1}=O\left(L^{3}\right)$. On the other hand, when the system is in one of the symmetry broken states, the length of the jam of the majority species is only diffusing. So it dissolves in a time of only $T_{2}=O\left(L^{2}\right)$, which is the lifetime of a symmetry broken state for $\alpha=\beta$. Thus, no symmetry breaking takes place in this case. Instead, for large $L$ a typical configuration is taken from a cycle, consisting of jams with diffusing length and of low density regions for both species. An average over many realizations leads to an approximately linear density profile as shown in Figure11 (lower left). Further, for $\alpha=\beta$ site $2(L)$ is occupied by $A$ particles for approximately half of a cycle length with probability $\alpha(1)$, leading to $\rho_{A}(2)=\alpha / 2$ and $\rho_{A}(L)=1 / 2$. For odd sides an analogous argument yields $\rho_{A}(1)=0$ and $\rho_{A}(L-1)=(1-\alpha) / 2$, which agrees well with Figure 1. Moreover, the formation and dissolution of boundary jams for the two species shows interesting temporal correlations [1].

It is also very interesting to compare these findings to the results of a mean-field treatment of the system. Performing a similar analysis as in [2] yields the same results for the steady state as the above analysis, except for the transition line $\alpha=\beta$. Along this line, mean field theory predicts a second symmetry-broken phase, where the density of $A(B)$ particles in the bulk is $\alpha_{1}(0)$ on even sites and $0\left(\alpha_{2}\right)$ on odd sites, with the constraint $\alpha_{1}+\alpha_{2}=\alpha$. This prediction corresponds to the disputed low-asymmetric phase of the original bridge model [2 4], where for the present model only the sum of the $A$ - and $B$-densities is fixed. In the present model, this phase does not exist as explained above and the mean-field prediction $\alpha_{1}+\alpha_{2}=\alpha$ is in clear contradiction to the observed density profile in Figure 1 (lower left).

In the present article, a two-species driven model with deterministic bulk behavior was investigated. The mechanism for spontaneous symmetry breaking in this model was described, leading to estimates for the relevant time scales in the broken phase and proving the existence of spontaneous symmetry-breaking without further assumptions on the rates. Along the transition line, the failure of a mean-field treatment, leading to the prediction of a second asymmetric phase, was explicitly demonstrated. In fact, the time evolution of the total density can be described for both the symmetric and the asymmetric phase and will be treated in a forthcoming publication [10].

\section{REFERENCES}

[1] Schütz G. M., J. Phys. A, 36 (2003) R339.

[2] Evans M. R., Foster D. P., Godrèche C. and Mukamel D., J. Stat. Phys., 80 (1995) 69.

[3] Clincy M., Evans M. R. and Mukamel D., J. Phys. A, 44 (2001) 9923.

[4] Arndt P. F., Heinzel T. and Rittenberg V., J. Stat. Phys., 90 (1998) 783.

[5] Godrèche C. et al., J. Phys. A, 28 (1995) 6.

[6] Levine E. and Willmann R. D., J. Phys. A, 37 (2004) 3333.

[7] Popkov V. and Schütz G. M., J. Stat. Mech.: Theor. Exp., P12004 (2004).

[8] Tóth B. and Valkó B., J. Stat. Phys., 112 (2003) 497.

[9] Schütz G., Phys. Rev. E, 47 (1993) 4265.

[10] Großkinsky S., Schütz G. M. and Willmann R. D., in preparation.

[11] Popkov V., J. Phys. A, 37 (2004) 1545. 